\title{
Structural study of coacervation in protein-polyelectrolyte complexes
}

\author{
S. Chodankar, ${ }^{1}$ V. K. Aswal, ${ }^{1}$ J. Kohlbrecher, ${ }^{2}$ R. Vavrin, ${ }^{2}$ and A. G. Wagh ${ }^{1}$ \\ ${ }^{1}$ Solid State Physics Division, Bhabha Atomic Research Centre, Mumbai-400 085, India \\ ${ }^{2}$ Laboratory for Neutron Scattering, ETH Zurich and Paul Scherrer Institut, CH-5232 Villigen PSI, Switzerland
}

(Received 3 June 2008; revised manuscript received 7 July 2008; published 17 September 2008)

\begin{abstract}
Coacervation is a dense liquid-liquid phase separation and herein we report coacervation of protein bovine serum albumin (BSA) in the presence of polyelectrolyte sodium polystyrene sulfonate (NaPSS) under varying solution conditions. Small-angle neutron scattering (SANS) measurements have been performed on above protein-polyelectrolyte complexes to study the structural evolution of the process that leads to coacervation and the phase separated coacervate as a function of solution $p \mathrm{H}$, protein-polyelectrolyte ratio and ionic strength. SANS study prior to phase separation on the BSA-NaPSS complex shows a fractal structure representing a necklace model of protein macromolecules randomly distributed along the polystyrene sulfonate chain. The fractal dimension of the complex decreases as $p \mathrm{H}$ is shifted away from the isoelectric point $(\sim 4.7)$ of BSA protein, which indicates the decrease in the compactness of the complex structure due to increase in the charge repulsion between the protein macromolecules bound to the polyelectrolyte. Concentration-dependence studies of the polyelectrolyte in the complex suggest coexistence of two populations of polyelectrolytes, first one fully saturated with proteins and another one free from proteins. Coacervation phase has been obtained through the turbidity measurement by varying $p \mathrm{H}$ of the aqueous solution containing protein and polyelectrolyte from neutral to acidic regime to get them to where the two components are oppositely charged. The spontaneous formation of coacervates is observed for $p \mathrm{H}$ values less than 4. SANS study on coacervates shows two length scales related to complex aggregations (mesh size and overall extent of the complex) hierarchically branched to form a larger network. The mesh size represents the distance between cross-linked points in the primary complex, which decreases with increase in ionic strength and remains the same on varying the proteinpolyelectrolyte ratio. On the other hand, the overall extent of the complex shows a similar structure irrespective of varying ionic strength and protein-polyelectrolyte ratio. A large fraction $(\sim 50 \%)$ of protein-polyelectrolyte complexes is also found to be free in the supernatant after the coacervation.
\end{abstract}

DOI: 10.1103/PhysRevE.78.031913

PACS number(s): 87.14.E-, 87.15.N-, 61.05.fg

\section{INTRODUCTION}

Polyelectrolytes interact strongly with proteins under suitable circumstances normally leading to phase separation: Liquid-liquid phase separation (coacervation) or solid-liquid phase separation (precipitation) [1]. Protein-polyelectrolyte complex leading to coacervation is a phenomenon in which a macromolecular aqueous solution separates into two immiscible liquid phases $[2,3]$. The denser phase, which is relatively concentrated in macromolecules, is called the coacervate and is in equilibrium with the relatively dilute macromolecular liquid phase [4]. Protein-polyelectrolyte interactions arise from the interaction between a threedimensional fixed and heterogeneously charged protein with a flexible charged chain strand [5]. They demonstrate the somewhat unusual effect of strong binding even when both species have the same net charge. This occurs due to patch binding between the charged polyelectrolyte with the opposite distribution of surface charges [6]. Coacervation is useful in a wide range of applications, which includes protein separation process [7-10], microencapsulation in drug delivery $[2,11]$, etc. It is also of biological significance as it is involved with protein-DNA interactions [12]. All of these applications of protein-polyelectrolyte-based complex coacervation depend on its structural details.

Protein-polyelectrolyte interactions is mainly governed by electrostatic interactions and hence by simple variation in $p \mathrm{H}$ $[6,13,14]$ or ionic strength $[15-17]$ can be used to tune these interactions. Usually, the charge on protein is strongly $p \mathrm{H}$ dependent. Thus, the aggregation of complexes leading to coacervation is expected to be most favored for the $p \mathrm{H}$ values when the net charge of the complexes becomes zero. Ionic strength screens the charges among the macromolecules and therefore can also play an important role to control the structure of coacervation. The hydrophobic interactions are also of high interest, where hydrophobic groups of the polyelectrolyte interact with the hydrophobic patches of the proteins during the complexation [2]. It is believed that at conditions favorable for protein-polyelectrolyte binding prior to coacervate phase formation, primary soluble complexes are formed $[18,19]$. These soluble complexes, on suitably varying solution conditions, undergo an aggregation that leads to coacervation [20].

There are various experimental methods used in characterizing the coacervation process. These include methods such as turbidity [21], capillary electrophoresis (CE) [17], rheology [22], light scattering [23], transmission electron microscopy (TEM) [24], and small-angle neutron scattering (SANS) [20]. The turbidity measurements provide the region of transition between solutions to coacervation phase. Capillary electrophoresis can be been used to obtain the information about the variation of the charge on the soluble complexes leading to coacervation. The viscoelastic property of the coacervate is obtained using rheology [25]. The structural evolution prior to coacervation had been frequently performed using dynamic light scattering $[17,26]$. It measures the hydrodynamic size of the complex, which can be used to 
correlate the binding of the components in the complex. However, it has limitations to study opaque systems as is the case of coacervate. TEM in principle can be used to study the microstructure of the coacervate, but this technique has the disadvantage of low contrast of electron for hydrogenous systems. SANS is a powerful technique to investigate the structure of such type of complexes in detail and also can be used for systems both prior to coacervation and in the coacervate. In particular, SANS can provide size, shape and arrangement at different length scales in these systems. However, no such studies are available on protein-polyelectrolyte systems in the literature. Herein, we report SANS study of structural evolution during coacervation of protein bovine serum albumin (BSA) in presence of polyelectrolyte sodium polystyrene sulfonate (NaPSS). The coacervation of this protein-polyelectrolyte complex is examined as a function of solution $p \mathrm{H}$, protein-polyelectrolyte ratio and ionic strength. Turbidimetric titration has been used to identify the transition boundary between soluble complexes and coacervates.

\section{EXPERIMENT}

BSA protein (Catalogue No. 05480, molecular weight 66.4 KDa) and NaPSS (Catalogue No. 43457-4, molecular weight $100 \mathrm{KDa}$ ) were purchased from Fluka. Samples for turbidity measurements were performed using a Jenway $6505 \mathrm{uv} / \mathrm{vis}$ spectrometer by titrating the mixture containing 2 wt. $\%$ protein and 0.5 wt. $\%$ NaPSS with dilute hydrochloric acid $(\mathrm{HCl})$. Samples for SANS experiments were prepared by dissolving known amount of BSA and NaPSS in a buffer solution of $\mathrm{D}_{2} \mathrm{O}$. The use of $\mathrm{D}_{2} \mathrm{O}$ as solvent instead of $\mathrm{H}_{2} \mathrm{O}$ provides better contrast for hydrogenous solute components in neutron experiments. Sample $p \mathrm{H}$ was adjusted in the range from 4 to 7.5 by using two buffer solutions of acetate buffer for $p \mathrm{H} \mathrm{4-6}$ and phosphate buffer for $p \mathrm{H} \mathrm{6-8.} \mathrm{The}$ ionic strength of the solution was kept at $0.5 \mathrm{M} \mathrm{NaCl}$ to minimize the interparticle interactions in the system. Smallangle neutron scattering experiments were performed on the SANS-I instrument at the Swiss Spallation Neutron Source, SINQ, Paul Scherrer Institut, Switzerland [27]. The mean wavelength of the incident neutron beam was $12 \AA$ with a wavelength resolution of approximately $10 \%$. The scattered neutrons were detected using two-dimensional $96 \mathrm{~cm}$ $\times 96 \mathrm{~cm}$ detector. The experiments were performed at three sample-to-detector distances of $1.6,4.5$, and $15 \mathrm{~m}$, respectively, to cover the data in the wave vector transfer $Q$ range from 0.006 to $0.2 \AA^{-1}$. The measured SANS data were corrected and normalized to a cross-sectional unit using BerSANS-PC data processing software [28]. The measurements on pure aqueous solution of protein and polyelectrolyte were performed at 2 and $0.5 \mathrm{wt}$. \% concentrations, respectively. The experiments for $p \mathrm{H}$ effect were carried out at $p \mathrm{H}$ values of $7.5,6.5$, and 5.5 on protein-polyelectrolyte system at a BSA/NaPSS molar ratio 6 as obtained by mixing 2 wt. \% BSA with 0.5 wt. $\%$ NaPSS. The samples for different protein-polyelectrolyte ratios were prepared by keeping fixed $2 \mathrm{wt} . \%$ protein concentration and varying the polyelectrolyte concentration in the range $0.25-0.5 \mathrm{wt}$. $\%$. All the coacervate samples were obtained by titrating the protein-polyelectrolyte solutions with dilute $\mathrm{HCl}$ to $p \mathrm{H} 4$. The different coacervates as a function of varying ionic strength $(0.1,0.15$, and $0.2 \mathrm{M})$ and protein-polyelectrolyte ratio $(r=6,9$, and 12$)$ have been studied. The temperature for all the measurements was kept fixed at $30{ }^{\circ} \mathrm{C}$.

\section{DATA ANALYSIS}

In small-angle neutron scattering one measures the coherent differential scattering cross section per unit volume $[d \Sigma / d \Omega(Q)]$ as a function of $Q$. The differential scattering cross section for different solution conditions are treated as follows.

(a) Protein solution. A system consisting of monodispersed interacting protein macromolecules, $d \Sigma / d \Omega(Q)$ can be expressed as [29]

$$
\frac{d \Sigma}{d \Omega}(Q)=N_{p} V_{p}^{2}\left(\rho_{p}-\rho_{s}\right)^{2}\left[\left\langle F(Q)^{2}\right\rangle+\langle F(Q)\rangle^{2}(S(Q)-1)\right]+B,
$$

where $N_{p}$ is the proteins number density and $V_{p}$ is the volume of the protein macromolecule. $\rho_{p}$ and $\rho_{s}$ are the scattering length density of the protein and the solvent, respectively. $F(Q)$ is the single particle form factor and $S(Q)$ is the interparticle structure factor. $B$ is a constant term that represents the incoherent scattering background, which is mainly due to hydrogen in the sample.

In the case of a solution with low protein concentration, having high salt concentration and $p \mathrm{H}$ close to the isoelectric point of the protein, $S(Q)$ can be approximated to unity as the interparticle interactions are minimized [30], and Eq. (1) for such system becomes

$$
\frac{d \Sigma}{d \Omega}(Q)=N_{p} V_{p}^{2}\left(\rho_{p}-\rho_{s}\right)^{2}\left\langle F(Q)^{2}\right\rangle+B
$$

The single particle form factor of the protein macromolecules in their native conformation has been calculated by treating them as prolate ellipsoids [31].

(b) Polyelectrolyte solution. The structure of polyelectrolyte sodium polystyrene sulfonate is fitted as a Gaussian chain conformation. In this case, scattering cross section is given as [32]

$$
\frac{d \Sigma}{d \Omega}(Q)=I_{0}\left[Q^{2} R_{g}^{2}-1+\exp \left(-Q^{2} R_{g}^{2}\right)\right] /\left(Q R_{g}\right)^{4}+B,
$$

where $R_{g}$ is the radius of gyration of the polyelectrolyte chain and $I_{0}$ is the forward scattering intensity. Equation (3) for higher $Q$ values $\left(Q R_{g}>1\right)$ reduces to the following scattering power law

$$
\frac{d \Sigma}{d \Omega}(Q) \sim \frac{1}{Q^{2}}+B
$$

(c) Protein-polyelectrolyte complexes. Protein-poly electrolyte complex in solution phase has been modeled as a fractal structure using the necklace model that assumes protein molecules randomly distributed along the polyelectrolyte chain. The cross section for such a system can be written as [33] 


$$
\frac{d \Sigma_{s}}{d \Omega}(Q) \sim P_{p}(Q) S_{f}(Q)+B,
$$

where $P_{p}(Q)$ denotes the normalized intraparticle structure factor of a single protein molecule, which for a spherical particle of radius $R$ is given by

$$
P_{p}(Q)=\left(\frac{3[\sin (Q R)-Q R \cos (Q R)]}{(Q R)^{3}}\right)^{2} .
$$

$S_{f}(Q)$ has been calculated using fractal structure for the necklace model of the protein-polyelectrolyte complex. The arrangement of proteins is assumed as fractal packing of spheres. In this case, $S_{f}(Q)$ is given as [34]

$$
\begin{aligned}
S_{f}(Q)= & 1+\frac{1}{(Q R)^{D}} \frac{D \Gamma(D-1)}{\left[1+(Q \xi)^{-2}\right]^{[(D-1) / 2]}} \\
& \times \sin \left[(D-1) \tan ^{-1}(Q \xi)\right],
\end{aligned}
$$

where $D$ is the fractal dimension of the protein distribution in space and $\xi$ the correlation length that is a measure of the extent of the complex. This structure factor in the intermediate $Q$ range $(1 / \xi<Q<1 / R)$ has the following power-law behavior:

$$
S_{f}(Q) \sim \frac{1}{Q^{D}} .
$$

The scattering cross section for phase separated coacervate is treated for a hierarchically branched structure with two length scales in two distinct $Q$ scales, low $Q$ region (Debye-Bueche behavior) and the high $Q$ region (OrnsteinZernike behavior). The structure factor for concentration fluctuations in the Ornstein-Zernike (OZ) region is given by [35]

$$
S_{L}(Q)=\frac{I_{L}(0)}{1+Q^{2} \zeta^{2}},
$$

where $\zeta$ is the correlation length of the concentration fluctuation representing mesh size (the cross-link distance among the primary complex) of the network and $I_{L}(0)$ is related to the cross-link density. Long wavelength concentration fluctuations in these systems often give rise to "excess scattering" in the low $Q$ region of SANS data. It has been suggested that long-range random inhomogeneities with correlation length many times larger than the radius of gyration of the dissolved particles are responsible for this excess scattering. If the spatial scale of density fluctuations due to the presence of inhomogenities is large compared to the correlation length $\zeta$, then the two contributions can be treated separately and added to give the total scattering cross section as $[36,37]$

$$
\frac{d \Sigma}{d \Omega}(Q)=S_{L}(Q)+S_{D}(Q),
$$

where $S_{L}(Q)$ is the Ornstein-Zernike $(\mathrm{OZ})$ function and the Debye-Bueche (DB) structure factor has the form of $S_{D}(Q)$ given by

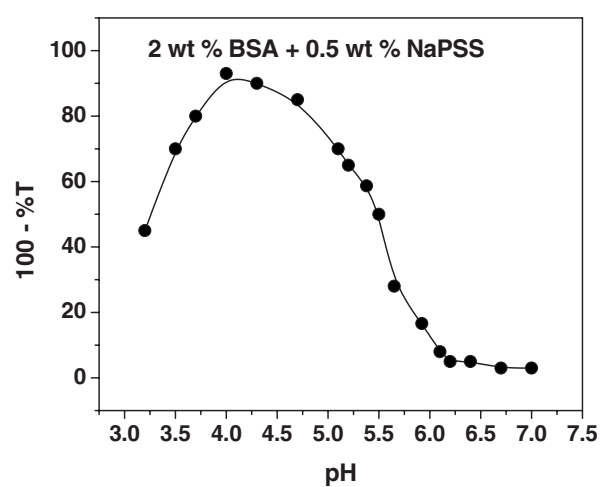

FIG. 1. Turbidity (100\% T, T is transmittance) data for $2 \mathrm{wt} . \%$ BSA in presence of $0.5 \mathrm{wt}$. $\% \mathrm{NaPSS}$ as a function of $p \mathrm{H}$ of the solution.

$$
S_{D}(Q)=\frac{I_{D}(0)}{\left(1+Q^{2} \Xi^{2}\right)^{2}},
$$

where $I_{D}(0)$ is the extrapolated structure factor at zero wave vector and $\Xi$ is the overall length of the inhomogenities.

\section{RESULTS AND DISCUSSION}

Turbidity measurements were performed on a mixture of protein BSA and polyelectrolyte NaPSS at fixed BSA/NaPSS ratio $(r=6)$ to examine the interaction of BSA and NaPSS as a function of $p \mathrm{H}$ and to identify the region of the coacervate phase separation. The concentrations of protein and polyelectrolyte were fixed at 2 and 0.5 wt. \%, respectively, and the $p \mathrm{H}$ of the solution decreased gradually from 7 to 3 by titrating the complex system with dilute $\mathrm{HCl}$. The measured turbidity data are shown in Fig. 1. It is observed that the BSA/ NaPSS system has about zero turbidity near $p \mathrm{H}$ value of 7 and remains relatively unchanged on decreasing the $p \mathrm{H}$ down to 6 . This suggests high solubility of the individual protein-polyelectrolyte complexes in aqueous solution. Further decrease in $p \mathrm{H}$ leads to increase in turbidity, which is an indication of some aggregation occurring between proteinpolyelectrolyte complexes. The aggregation of complexes can be understood based on the change in electrostatic interaction as a function of $p \mathrm{H}$ among protein and polyelectrolyte. BSA protein has an isoelectric point at $p \mathrm{H} 4.7$ and below which it has a net positive charge. Thus at low $p \mathrm{H}$ values charge neutralization between protein and polyelectrolyte in the complexes increases the propensity of aggregation of these complexes and hence the turbidity increases with decrease in $p \mathrm{H}$ value [21]. The increase in turbidity continues up to $p \mathrm{H} 4$, where a maximum in turbidity is found, below which it decreases. The fall in solution turbidity below $p \mathrm{H} 4$ is due to the formation of coacervate, and which separates to the bottom.

Figure 2 shows SANS data for aqueous solution of BSA protein and NaPSS polyelectrolyte at $p \mathrm{H}$ 5.5. SANS data for 2 wt. \% BSA solution [Fig. 2(a)] are fitted considering a prolate ellipsoidal shape [Eq. (2)] of the protein macromolecules. The analysis gives the semimajor and semiminor axes values of the protein macromolecules as $70.5 \pm 5.1 \AA$ and 

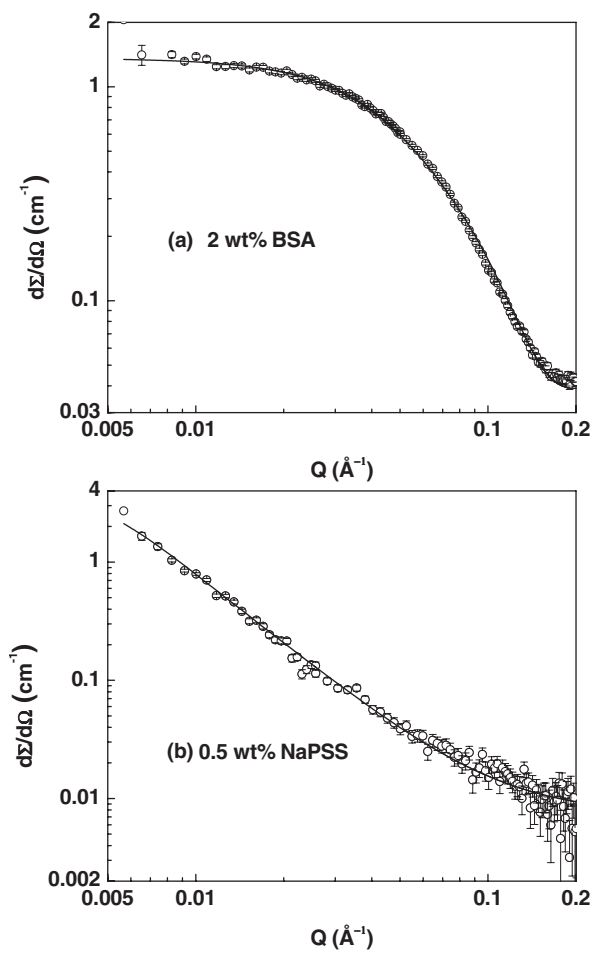

FIG. 2. SANS data on aqueous solution of (a) 2 wt. \% BSA and (b) 0.5 wt. $\%$ NaPSS.

$22.1 \pm 0.8 \AA$, respectively, which is similar to that reported in the literature $[34,38,40]$. SANS data for aqueous solution of 0.5 wt. \% NaPSS [Fig. 2(b)] are fitted using a Gaussian chain conformation [Eq. (3)] of the polyelectrolyte [41,42]. The value of radius of gyration is found to be $385.3 \pm 19.5 \AA$.

Figure 3 shows the SANS data on the proteinpolyelectrolyte complex obtained from mixing of pure protein [Fig. 2(a)] and polyelectrolyte [Fig. 2(b)]. The features of the scattering data of the complex are significantly differ-

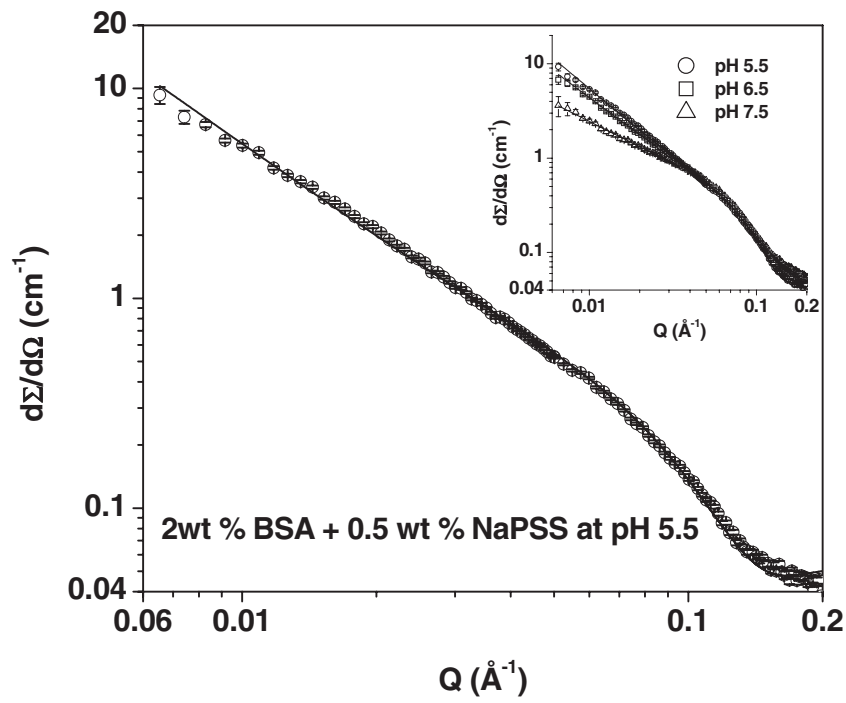

FIG. 3. SANS data for $2 \mathrm{wt} \%$ BSA in presence of $0.5 \mathrm{wt}$. $\%$ $\mathrm{NaPSS}$ at $p \mathrm{H}$ 5.5. Inset shows the SANS data for the same sample at various $p \mathrm{H}$ values.
TABLE I. Fitted parameters of SANS analysis for $2 \mathrm{wt}$ \% BSA in presence of $0.5 \mathrm{wt}$. $\%$ NaPSS with varying solution $p \mathrm{H}$. Data are fitted for a fractal structure of protein molecules randomly distributed along the polyelectrolyte chain.

\begin{tabular}{lcc}
\hline \hline$p \mathrm{H}$ & $\begin{array}{c}\text { Radius } \\
R(\AA)\end{array}$ & $\begin{array}{c}\text { Fractal dimension } \\
D\end{array}$ \\
\hline 5.5 & $27.2 \pm 1.1$ & $1.65 \pm 0.01$ \\
6.5 & $26.7 \pm 0.7$ & $1.47 \pm 0.01$ \\
7.5 & $26.8 \pm 0.7$ & $1.08 \pm 0.01$ \\
\hline \hline
\end{tabular}

ent as compared to those of pure protein and polyelectrolyte solutions. One of the interesting characteristics in Fig. 3 is the linearity of the scattering profile on a logarithmic scale in the lower $Q$ region. This is an indication of fractal structure of the protein-polyelectrolyte complex [34]. The fractal structure is modeled on the basis of a necklace model considering binding of proteins along the polyelectrolyte chain [Eq. (7)]. The scattering from the protein-polyelectrolyte is dominated by the bound proteins around in the complex and the scattering from the polyelectrolyte is neglected. This is because the scattering from the polyelectrolyte is quite low as compared to that from the complex. This is further expected to be reduced for the polyelectrolyte in the complex as the scattering contrast will decrease on expansion [34,39]. In this model the higher cutoff of the linearity of the scattering data is related to the size of the protein attached to the polyelectrolyte and the lower cutoff corresponds to the overall size of the protein-polyelectrolyte complex. In the measured $Q$ range, the position of low $Q$ cutoff is not observed. The lowest $Q$ value is $0.006 \AA^{-1}$, which suggests that the protein-polyelectrolyte complex has a size $\xi$ larger than $2 \pi / Q_{\min }$ (i.e., $1000 \AA$ ). The slope of the scattering data gives the value of the fractal dimension $D$ of the complex. The analysis gives the fractal dimension of the complex as $1.65 \pm 0.01$ and the individual protein molecule bounded to the polyelectrolyte has a size of $27.2 \pm 1.1 \AA$. This size of the protein molecule in the complex is found to be smaller to the averaged size of the native protein $(32 \AA)$, which may be due to unfolding of certain patches of protein on binding with NaPSS [43].

SANS data for effect of varying $p \mathrm{H}$ on a proteinpolyelectrolyte complex at fixed BSA/NaPSS ratio $(r=6)$ is shown in the inset of Fig. 3. The charge on both the protein and the polyelectrolyte is negative for the $p \mathrm{H}$ values above isoelectric point (4.7) of the BSA protein. The scattering cross-section of the complex in the low $Q$ region decreases with increasing $p \mathrm{H}$. The slope of the linear region also decreases suggesting decrease in the fractal dimension of the complex with increase in $p \mathrm{H}$. This can be understood on the basis of the increase in electrostatic repulsion between proteins within the complex as the charge on the protein increases with $p \mathrm{H}$. This leads to decrease in compactness of the complex as the complex prefers to be in linear extended structures to minimize the electrostatic interactions. The fitted parameters in these systems are given in Table I.

SANS data on protein-polyelectrolyte complexes at various BSA/NaPSS ratios and at a $p \mathrm{H}$ of 6.5 are shown in Fig. 


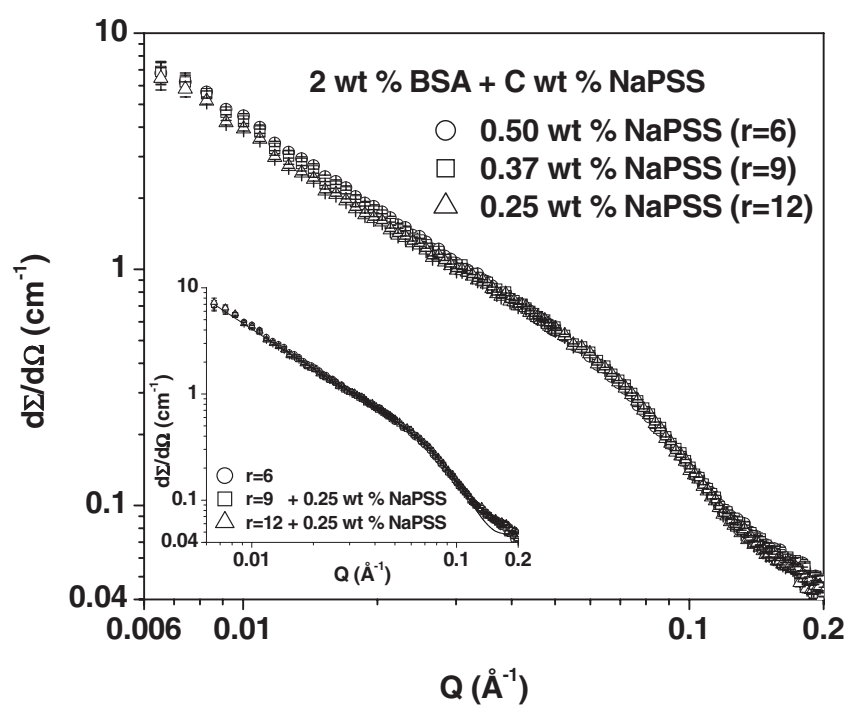

FIG. 4. SANS data for various BSA/NaPSS ratios obtained from 2 wt. \% BSA at different NaPSS concentration (0.25-0.5 wt. \%). Inset shows the SANS data for the various ratios summed by the SANS data for the free polyelectrolyte concentration.

4. The protein-polyelectrolyte ratio is varied keeping the protein concentration fixed at $2 \mathrm{wt} . \%$ and changing the polyelectrolyte concentration. The data are shown for proteinpolyelectrolyte ratios of 6,9 , and 12 . It is interesting to note that SANS profiles for various BSA/NaPSS ratios are almost similar. This is possible if the structure of the complex is unaffected by the increase in ratio. That is the complex structure is independent of protein and polyelectrolyte concentrations. This suggests protein macromolecules do not distribute uniformly to all the polyelectrolytes instead they prefer to form an equilibrated complex with only limited polyelectrolytes depending on the number of protein macromolecules. We have not observed any significant change when the BSA/ NaPSS ratio is increased from $r=6$ to 12 , perhaps due to the fact that at $r=6$ more than $50 \%$ of polyelectrolytes are not forming a complex with the protein macromolecule. If it is so, the data do not show significant change in Fig. 4 as the scattering from free polyelectrolytes is very small [Fig. 2(b)], about one order less than that from the complex structure (Fig. 3). This is further clarified in the inset of Fig. 4 where on addition of 0.25 wt. \% NaPSS with $r=12$ (2 wt. \% $\mathrm{BSA}+0.25 \mathrm{wt}$. \% NaPSS) the data looks similar to that of $r=6$ ( 2 wt. \% BSA +0.5 wt. \% NaPSS). Figure 5 shows the effect of addition of protein on fixed concentration of polyelectrolyte $(0.25 \mathrm{wt} . \%)$. These data suggest to our argument of coexistence of two populations of polyelectrolytes, first one fully saturated with proteins and another one free from proteins. The fact that the scattering can be scaled to the factors by which the ratio of protein to polyelectrolyte is increased in the complex (inset of Fig. 5), suggests increase in the number of saturated complexes instead of change in the structure of the complex.

The SANS data from coacervate samples are shown in Figs. 6-8. All the coacervates were prepared by lowering the $p \mathrm{H}$ to 4 , where the turbidity shows a maximum (Fig. 1). To accelerate the process of phase separation, the samples were

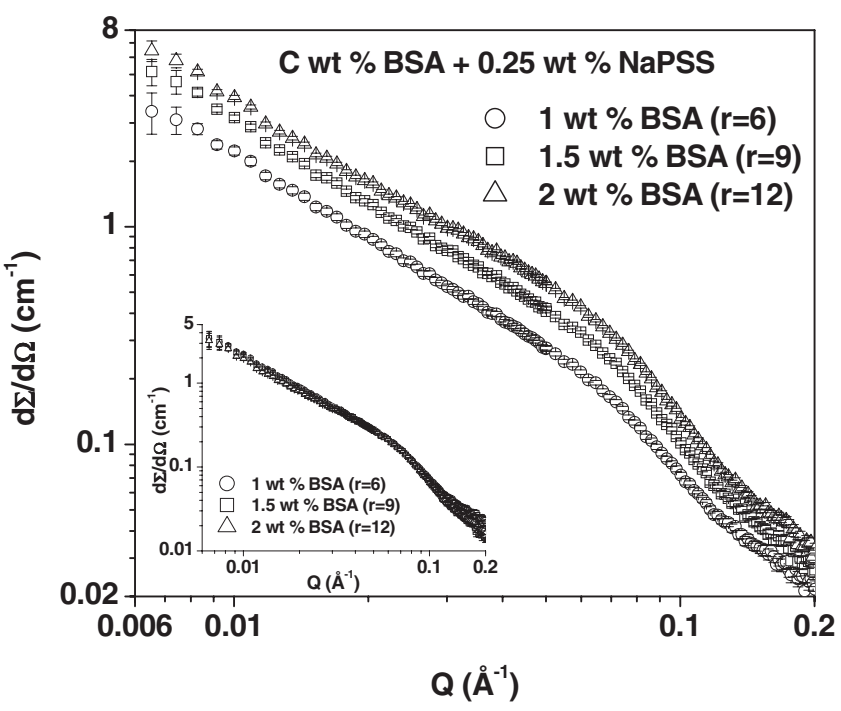

FIG. 5. SANS data for various BSA/NaPSS ratios obtained from varying BSA concentrations $(1,1.5$, and $2 \mathrm{wt} . \%)$ at $0.25 \mathrm{wt} . \%$ NaPSS concentration. Inset shows the SANS data for the various ratios scaled by their protein concentration.

centrifuged at $4500 \mathrm{rpm}$ for 30 minutes after which the supernatants were carefully separated from the coacervate phase and the coacervates were placed in aluminum foil for SANS measurements. Figure 6 shows the SANS data for a phase separated coacervate sample as prepared from a protein-polyelectrolyte complex having $2 \mathrm{wt}$ \% BSA and 0.5 wt. $\%$ NaPSS. For comparison SANS data from soluble protein-polyelectrolyte complex prior to coacervation at $p \mathrm{H}$ 5.5 are also shown in Fig. 6. It is observed that soluble protein-polyelectrolyte complex and their coacervate shows very different scattering profiles. In particular, in the low $Q$ region coacervate sample shows a strong buildup in scattering intensity. This is expected due to high density of proteinpolyelectrolyte complexes in the coacervate. Unlike soluble protein-polyelectrolyte complex, which shows a linear behavior on a logarithmic scale representing a fractal structure, coacervate sample has a different $Q$ dependence. The SANS data for coacervate samples have been fitted by two scattering laws, first one representing the mesh size of proteinpolyelectrolyte complex and another one for the size of the

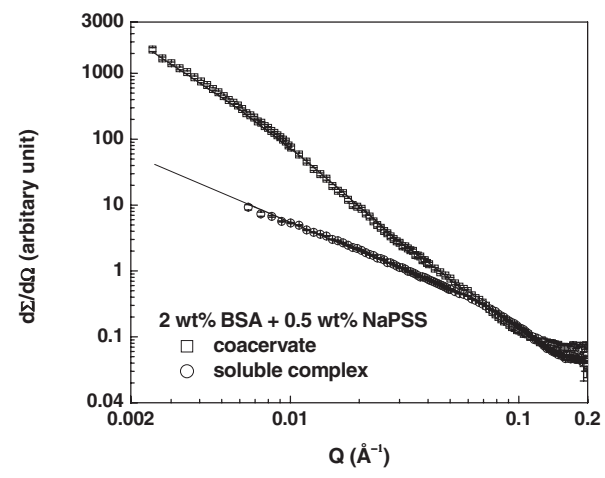

FIG. 6. SANS data for the coacervate and proteinpolyelectrolyte complex prepared from $2 \mathrm{wt}$. \% BSA and $0.5 \mathrm{wt}$. \% NaPSS concentration. 


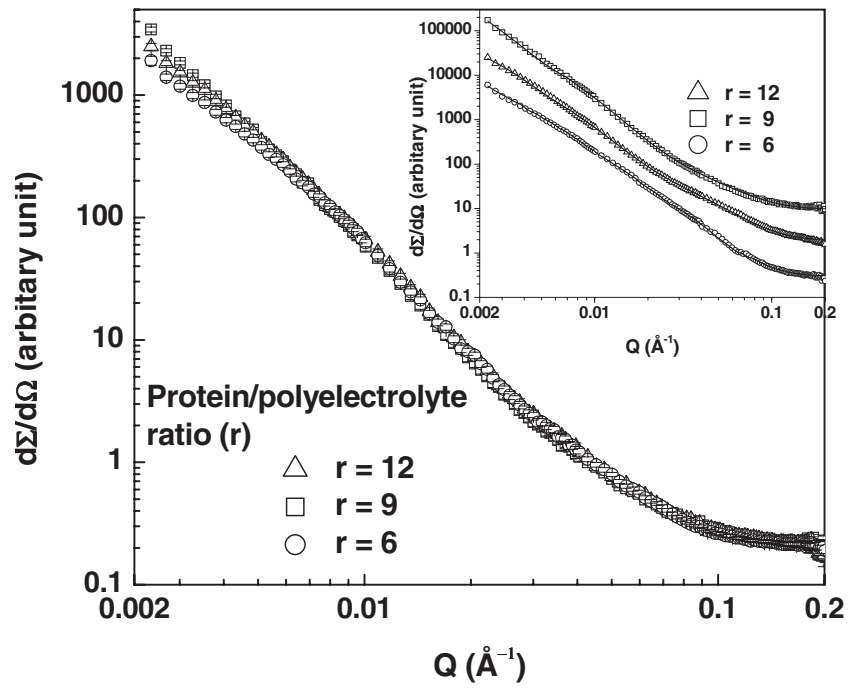

FIG. 7. SANS data for the coacervate prepared at various BSA/ NaPSS ratios. Inset shows the fitted curves, which are vertically shifted for clarity.

complex in the aggregates. Coacervation is considered to be a liquid-liquid phase separation, where the aggregation of the smaller inhomogeneities (protein-polyelectrolyte complexes) gives rise to larger aggregates that are solidlike in nature. Thus, coacervates consist of both liquidlike and solidlike properties. The length scale of coacervate phase representing mesh size within the protein-polyelectrolyte complex is fitted considering the Ornstein-Zernike (OZ) equation, which is adopted in the case of, liquid rather than solid. Whereas, the individual complexes are similar to a frozen solidlike system, which has, been fitted considering the Debye-Bueche (DB) equation. The analysis using Eq. (9) that takes account of both the $\mathrm{OZ}$ and $\mathrm{DB}$ contributions, gives the mesh size as $82.8 \AA$ and the complex size as $436.4 \AA$

The SANS data for the coacervates at different BSA/ NaPSS ratios prepared from $2 \mathrm{wt}$. $\%$ BSA and on varying

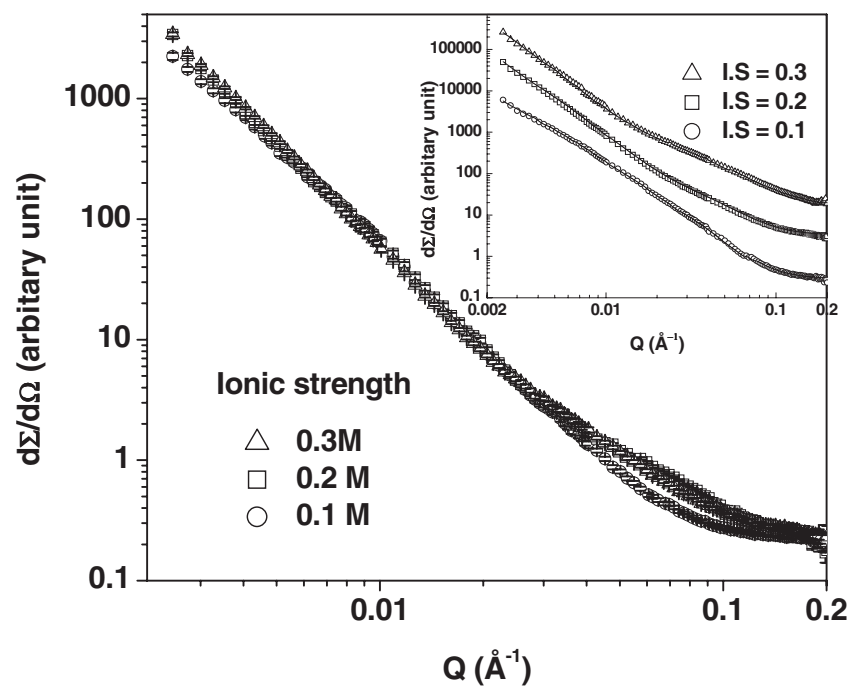

FIG. 8. SANS data for the coacervate prepared at fixed BSA/ NaPSS ratios $(r=6)$ and at various ionic strengths. Inset shows the fitted curves, which are vertically shifted for clarity.
TABLE II. Fitted parameters of SANS analysis for phase separated coacervate systems obtained for various proteinpolyelectrolyte ratios and at fixed $0.1 \mathrm{M}$ ionic strength.

\begin{tabular}{lcc}
\hline \hline$[\mathrm{BSA} / \mathrm{NaPSS}]$ & $\begin{array}{c}\text { Mesh size } \\
\zeta(\AA)\end{array}$ & $\begin{array}{c}\text { Complex size } \\
\Xi(\AA)\end{array}$ \\
\hline 6 & $82.8 \pm 4.7$ & $436.5 \pm 9.1$ \\
9 & $80.4 \pm 4.5$ & $447.8 \pm 10.3$ \\
12 & $83.4 \pm 4.8$ & $452.2 \pm 11.5$ \\
\hline \hline
\end{tabular}

NaPSS concentration (0.5-0.25 wt. \%) are shown in Fig. 7. The data do not show any significant change, indicating no change in the coacervate structure with variation in the BSA/ NaPSS ratio, which is similar to that observed for primary soluble complexes (Fig. 4). The inset of Fig. 7 shows the fitted data after vertically shifting for clarity. The parameters of the analysis are given in Table II. The mesh size and the complex size in the coacervates have similar values within the error bar for different BSA/NaPSS ratios. Figure 8 shows the effect of ionic strength on the coacervate structure having protein-polyelectrolyte ratio of 6 . In this case SANS data show a change in the higher $Q$ region unlike to that in Fig. 7. The parameters of the analysis are given in Table III. It is found that the mesh size decreases with increase in ionic strength. However, the complex size in the coacervate remains similar. This suggests different distribution of proteins in the protein-polyelectrolyte complex as controlled by the ionic strength. When ionic strength is high, charged proteins can come closer, and this leads to decrease in mesh size.

Figure 9 shows the SANS data for the supernatant of the coacervate sample at BSA/NaPSS ratio of 6 and compared with data from soluble protein-polyelectrolyte complex prior to coacervation at $p \mathrm{H}$ 5.5. The scattering from the supernatant with respect to the soluble complex as expected decreases. However, it is significantly large suggesting that large fraction of protein-polyelectrolyte complexes is still present in the system, which do not participate to form coacervate. The features of the scattering profile of supernatant also change significantly as compared to the soluble complex prior to coacervation. There is buildup of scattering cross section in the low as well as high $Q$ region. The buildup in the low $Q$ region suggests the increase in the fractal dimension of the protein-polyelectrolyte complex in the supernatant. The fractal dimension increases as perhaps at coacervation protein molecules in the complex experience less repulsion, caused by the charge neutralization on the protein

TABLE III. Fitted parameters of SANS analysis for phase separated coacervate systems obtained for various ionic strengths and at a fixed protein-polyelectrolyte ratio of 6 .

\begin{tabular}{lcc}
\hline \hline $\begin{array}{l}\text { Ionic strength } \\
(\mathrm{M})\end{array}$ & $\begin{array}{c}\text { Mesh size } \\
\zeta(\AA)\end{array}$ & $\begin{array}{c}\text { Complex size } \\
\Xi(\AA)\end{array}$ \\
\hline 0.1 & $82.8 \pm 4.7$ & $436.5 \pm 9.1$ \\
0.2 & $59.8 \pm 3.6$ & $450.3 \pm 10.6$ \\
0.3 & $53.6 \pm 3.3$ & $445.6 \pm 10.1$ \\
\hline
\end{tabular}




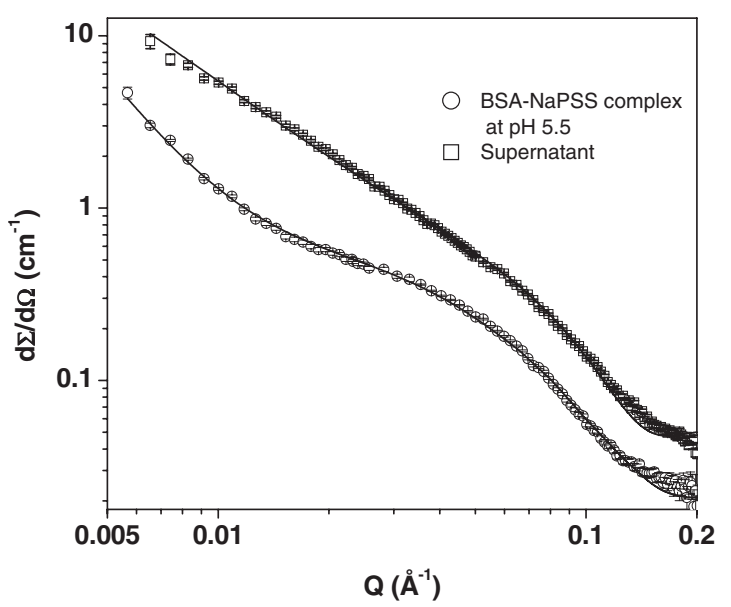

FIG. 9. SANS data on supernatant of phase separated coacervate obtained from mixture of $2 \mathrm{wt}$. \% BSA in presence of $0.5 \mathrm{wt} . \%$ NaPSS at $p \mathrm{H} 4$. Data of $2 \mathrm{wt} . \%$ BSA in presence of $0.5 \mathrm{wt} . \%$ NaPSS at $p \mathrm{H} 5.5$ prior to coacervation are also shown for comparison.

molecule due to the strong binding of the oppositely charged polyelectrolyte. On the other hand, the buildup at the high $Q$ region relates to the higher size of the protein molecule bound to the complex. This is possible if polyelectrolytes and proteins carry opposite charges at coacervation, which enhances the direct electrostatic binding of the protein without any deformation. The fractal dimension of the complex increases from 1.65 for a soluble complex to 2.67 for a supernatant followed by the increase in the average size of the protein in the complex from 27 to $33.5 \AA$ for these two systems. The normalization of the SANS data prior to coacer- vation with the supernatant, gives about $50 \%$ of the complexes still present in the supernatant after the coacervation. This result is also verified by the water evaporation technique that measures the masses of the coacervate and the supernatant before and after full evaporation of water.

\section{CONCLUSIONS}

The structural studies of BSA-NaPSS complexes prior to coacervation and on coacervates as a function of $p \mathrm{H}$, protein-polyelectrolyte ratio and ionic strength have been performed using SANS. The coacervation is observed by decreasing the $p \mathrm{H}$ of the protein-polyelectrolyte solution, where the turbidity shows a maximum. SANS study on the protein-polyelectrolyte complexes prior to coacervation represents a fractal structure of the complex, consisting of binding of protein macromolecules to the polyelectrolyte chain. The fractal dimension of the complex increases as $p \mathrm{H}$ is approached towards the isoelectric point, which suggests increase in compactness of the complex structure as a result of decrease in the net charge on the protein molecule. The phase separated coacervate samples show two length scales representing aggregation of complexes (i.e., mesh size and overall size of the complex in aggregation) hierarchically branched to form a larger network. Supernatant of the coacervation still contains a large fraction of free protein-polyelectrolyte complexes after the coacervation.

\section{ACKNOWLEDGMENTS}

This work is based on SANS experiments performed at the Swiss Spallation Neutron Source SINQ, Paul Scherrer Institut, Villigen, Switzerland.
[1] H. G. B. de Jong, in Colloid Science, edited by H. R. Kruyt (Elsevier, Amsterdam, 1949), Vol. II.

[2] C. L. Cooper, P. L. Dubin, A. B. Kayitmazer, and S. Turksen, Curr. Opin. Colloid Interface Sci. 10, 52 (2005).

[3] J. L. Doublier, C. Garnier, D. Renard, and C. Sanchez, Curr. Opin. Colloid Interface Sci. 5, 202 (2000).

[4] H. B. Bohidar, P. L. Dubin, P. R. Majhi, C. Tribet, and W. Jaeger, Biomacromolecules 6, 1573 (2005).

[5] A. Tsuboi, T. Izumi, M. Hirata, J. Xia, P. L. Dubin, and E. Kokututa, Langmuir 12, 6295 (1996).

[6] R. K. Hallberg and P. L. Dubin, J. Phys. Chem. B 102, 8629 (1998).

[7] E. M. Baskin, B. I. Shklovskii, and G. V. Zilberstein, Physica A 317, 313 (2003).

[8] Y. Wang, J. Y. Gao, and P. L. Dubin, Biotechnol. Prog. 12, 356 (1996).

[9] A. Cifuentes, H. Poppe, J. C. Kraak, and F. B. Erim, J. Chromatogr., B: Biomed. Sci. Appl. 681, 21 (1996).

[10] F. M. Menger and B. M. Sykes, Langmuir 14, 4131 (1998).

[11] A. B. Kayitmazer, D. Shaw, and P. L. Dubin, Macromolecules 38, 5198 (2005).

[12] J. Xia and P. L. Dubin, Macromolecular Complexes in Chemistry and Biology (Springer-Verlag, Berlin, 1994).
[13] C. Schmitt, C. Sanchez, S. Desobry-Banon, and J. Hardy, Crit. Rev. Food Sci. Nutr. 38, 689 (1998).

[14] K. Yoshida and P. L. Dubin, Colloids Surf., A 147, 161 (1999).

[15] T. Sato, K. W. Mattison, P. L. Dubin, M. Kamachi, and Y. Morishima, Langmuir 14, 5430 (1998).

[16] Y. Wang, K. Kimura, P. L. Dubin, and W. Jaeger, Macromolecules 33, 3324 (2000)

[17] E. Seyrek, P. L. Dubin, C. Tribet, and E. A. Gamble, Biomacromolecules 4, 273 (2003).

[18] J. M. Park, B. B. Muhoberac, P. L. Dubin, and J. Xia, Macromolecules 25, 290 (1992).

[19] J. Y. Gao, P. L. Dubin, and B. B. Muhoberac, J. Phys. Chem. B 102, 5529 (1998).

[20] J. Gummel, F. Boue, B. Deme, and F. Cousin, J. Phys. Chem. B 110, 24837 (2006).

[21] K. Kaibara, T. Okazaki, H. B. Bohidar, and P. L. Dubin, Biomacromolecules 1, 100 (2000).

[22] R. Borrega, C. Tribet, and R. Audebert, Macromolecules 32, 7798 (1999).

[23] Y. Li, K. W. Mattison, P. L. Dubin, H. A. Havel, and S. L. Edwards, Biopolymers 38, 527 (1995).

[24] F. M. Menger and B. M. Skyes, Langmuir 16, 9113 (2000).

[25] F. Weinbreck and R. H. W. Wientjes, J. Rheol. 48, 1215 
(2004).

[26] S. Rosenfeldt, A. Wittemann, M. Ballauff, E. Breininger, J. Bolze, and N. Dingenouts, Phys. Rev. E 70, 061403 (2004).

[27] J. Kohlbrecher and W. Wagner, J. Appl. Crystallogr. 33, 804 (2000).

[28] U. Keiderling, Appl. Phys. A: Mater. Sci. Process. 74, S1455 (2002).

[29] J. B. Hayter and J. Penfold, Colloid Polym. Sci. 261, 1022 (1983).

[30] S. Chodankar and V. K. Aswal, Phys. Rev. E 72, 041931 (2005).

[31] J. S. Pedersen, Adv. Colloid Interface Sci. 70, 171 (1997).

[32] P. Debye, J. Phys. Colloid Chem. 51, 18 (1947).

[33] J. Teixeira, J. Appl. Crystallogr. 21, 781 (1988).

[34] S. H. Chen and J. Teixeira, Phys. Rev. Lett. 57, 2583 (1986).

[35] P. G. De Gennes, Scaling Concepts in Polymer Physics (Cor- nell University Press, Ithaca, NY, 1985).

[36] B. Mohanty, V. K. Aswal, J. Kohlbrecher, and H. B. Bohidar, J. Polym. Sci., Part B: Polym. Phys. 44, 1653 (2006).

[37] P. Debye and A. M. Bueche, J. Appl. Phys. 20, 518 (1949).

[38] S. Chodankar, V. K. Aswal, J. Kohlbrecher, R. Vavrin, and A. G. Wagh, Phys. Rev. E 77, 031901 (2008).

[39] S. Chodankar, V. K. Aswal, J. Kohlbrecher, R. Vavrin, and A. G. Wagh, J. Phys.: Condens. Matter 19, 326102 (2007).

[40] S. F. Santos, D. Zanette, H. Fischer, and R. Itri, J. Colloid Interface Sci. 262, 400 (2003).

[41] M. D. Carbajal-Tinoco, R. Ober, I. Dolbnya, W. Bras, and C. E. Williams, J. Phys. Chem. B 106, 12165 (2002).

[42] T. J. Taylor and S. S. Stivala, J. Polym. Sci., Part B: Polym. Phys. 41, 1263 (2003).

[43] F. Cousin, J. Gummel, D. Ung, and F. Boue, Langmuir 21, 9675 (2005). 